\title{
Benefits of Weak Disorder in One-Dimensional Topological Superconductors
}

\author{
Arbel Haim ${ }^{1}$ and Ady Stern ${ }^{2}$ \\ ${ }^{1}$ Walter Burke Institute for Theoretical Physics and the Institute for Quantum Information and Matter, \\ California Institute of Technology, Pasadena, California 91125, USA \\ ${ }^{2}$ Department of Condensed Matter Physics, Weizmann Institute of Science, Rehovot 7610001, Israel
}

(Received 13 September 2018; published 29 March 2019)

\begin{abstract}
Majorana bound states are zero-energy modes localized at the ends of a one-dimensional (1D) topological superconductor. Introducing disorder usually increases the Majorana localization length, until eventually inducing a topological phase transition to a trivial phase. In this Letter, we show that in some cases weak disorder causes the Majorana localization length to decrease, making the topological phase more robust. Increasing the disorder further eventually leads to a change of trend and to a phase transition to a trivial phase. Interestingly, the transition occurs at $\xi_{0} \gg l$, where $l$ is the disorder mean free path, and $\xi_{0}$ is the localization length in the clean limit. Our results are particularly relevant to $1 \mathrm{D}$ topological superconductors formed in planar Josephson junctions.
\end{abstract}

DOI: 10.1103/PhysRevLett.122.126801

Introduction.-Understanding the effect of unavoidable disorder on topological superconductivity is of great interest. Of particular interest is its effect on the localization length of the zero-energy Majorana bound states (MBSs) and the critical strength for transition to a trivial state.

Effects of disorder on a spinless single-channel $p$-wave superconductor [1,2] - the canonical model for topological superconductivity (TSC) [3-7]-were previously studied [8-13]. Disorder was found to increase the Majorana localization length $\xi$ according to $1 / \xi=$ $1 / \xi_{0}-1 / 2 l$, with $\xi_{0}$ being the localization length (or coherence length) in the clean limit, and $l$ being the impurity-induced mean free path [9]. At the critical value $l_{\mathbf{c}}=\xi_{0} / 2$, the localization length diverges leading to a phase transition to a trivial phase. Accordingly, the critical mean free time $\tau_{\mathbf{c}}$ is determined by the excitation gap of the clean system $\tau_{\mathbf{c}}^{-1}=2 E_{\text {gap }}$ [14].

For a multichannel 1D system [15-19], at weak-enough disorder the behavior is similar to the single-channel case with monotonically increasing $\xi$. For stronger disorder, multiple transitions between trivial and topological occur at $l_{\mathbf{c}}^{(n)}=n \xi_{0} /(N+1)$, with $N$ the number of channels [16,17].

In this Letter, we study the effect of disorder on a novel realization of a 1D topological superconductor: a planar Josephson junction (JJ) implemented in a Rashba twodimensional electron gas (2DEG) and subject to in-plane magnetic field [20-23] (see Fig. 1). We find that in this system, weak potential disorder causes $\xi$ to decrease [see Fig. 1(b)]. For strong disorder, the trend eventually reverses, and the localization length increases back until finally diverging at the transition to the trivial phase. Importantly, this transition occurs at a critical disorder strength $\tau_{\mathbf{c}}^{-1}$, which is typically much larger than the gap of the clean system.
Studying a general low-energy model for a multichannel TSC, we show that disorder can cause $\xi$ to increase or decrease, depending on the relative phases of the pairing potentials in different channels and the structure of the interchannel impurity scattering (see also Fig. 2). Scattering between modes of equal-phase pairing potential increases the "effective" pairing gap, while scattering between modes of opposite-phase potentials decreases the effective gap. Because of the $p$-wave nature of the pairing within each
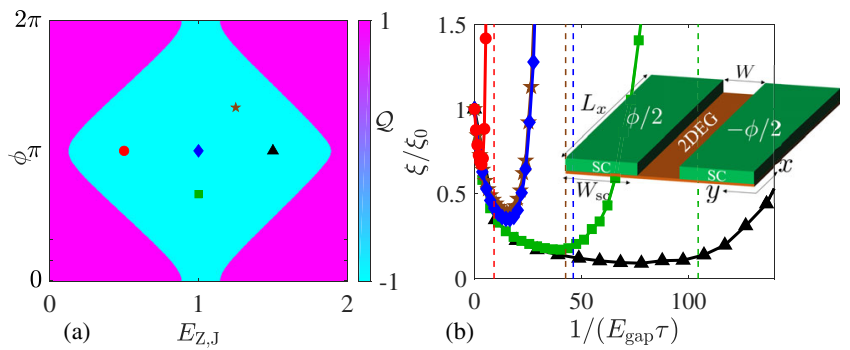

FIG. 1. (a) Phase diagram of the planar Josephson junction Eq. (1) in the clean limit. In the topological phase $(\mathcal{Q}=-1)$, the system supports zero-energy MBSs at each end of the junction. (b) The Majorana localization length $\xi$ versus the disorderinduced inverse mean free time $\tau^{-1}$ for different points inside the topological phase [see markers in (a)]. At weak disorder, $\xi$ decreases with disorder. For stronger disorder, $\xi$ increases until eventually diverging at the phase transition to the trivial phase. Here, $\xi$ is averaged over 100 disorder realizations. The system parameters are $E_{\mathrm{SO}}=m_{\mathrm{e}} \alpha^{2} / 2=1, \Delta_{0}=1, \mu_{\mathbf{J}}=\mu_{\mathrm{SC}}=2.5$, $E_{\mathbf{Z}, \mathrm{SC}}=0, l_{\mathrm{SO}}=1 / m_{\mathbf{e}} \alpha=0.2 W, W_{\mathrm{SC}}=W$. In (b), $\tau$ is normalized by the overall gap in the clean system $E_{\text {gap }}$, which is $0.032,0.032,0.022,0.008$, and 0.004 for the red, brown, blue, green, and black plots, respectively. Similarly, $\xi$ is normalized by that of the clean system $\xi_{0}$, which is $24 W, 34 W, 36 W, 93 W$, and $194 W$, in the same order. 


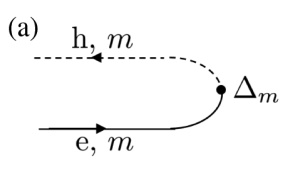

(b)

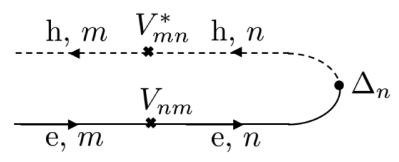

FIG. 2. (a) Andreev reflection (AR) in the $m$ th channel. (b) With disorder, the electron can first scatter to the $n$th channel, perform $\mathrm{AR}$, and then scatter back to the $m$ th channel.

channel, intrachannel backscattering always decreases the effective gap and delocalizes the MBS.

We find that the enhancement of localization by weak disorder in the planar $\mathrm{JJ}$ is related to the structure of the low-energy excitations confined to the junction. The excitations carry a longitudinal momentum $k_{x}$. The spectrum is gapped, and the smallest gap is at large $k_{x}$, close the Fermi momenta of the 2DEG [21]. At these $k_{x}$ 's spin-orbit coupling (SOC) dominates over the Zeeman field, causing the spins of opposite-momenta modes in each channel to be oppositely polarized, thereby suppressing the detrimental intrachannel backscattering [24]. Consequently, disorder effectively increases the gap of the large-momentum channels. In contrast, at small $k_{x}$, the Zeeman field dominates over SOC, allowing for intrachannel backscattering, which decreases the effective gap. The smallest of the gaps determines $\xi$. Weak disorder then increases the large-momentum gap and enhances localization. As disorder is increased, the trend changes when the gaps at small and large momentum become equal (see also Fig. 3).

We begin with a numerical analysis of the dependence of $\xi$ on disorder in a planar JJ. We then consider a low-energy model of a multichannel TSC. Finally, we construct a simplified model of the planar JJ, which qualitatively reproduces the numerical results.

Numerical analysis of the planar Josephson junction.The planar JJ consists of two conventional superconductors in proximity to a Rashba-spin-orbit-coupled 2DEG [23]. The superconductors are separated by a distance $W$ and are of length $L_{x}$ in the $x$ direction; see Fig. 1. As shown theoretically [20,21], by applying an in-plane magnetic field and controlling the phase bias, the junction can realize a 1D TSC. Experimental evidence for a TSC has been recently reported $[25,26]$.

In the presence of impurity-potential disorder, the system's Hamiltonian is

$$
\begin{aligned}
\mathcal{H}= & \left(-\frac{\nabla^{2}}{2 m_{\mathrm{e}}}-\mu(y)+U(x, y)-i \alpha\left(\sigma_{y} \partial_{x}-\sigma_{x} \partial_{y}\right)\right) \tau_{z} \\
& +E_{\mathrm{Z}}(y) \sigma_{x}+\operatorname{Re}[\Delta(y)] \tau_{x}-\operatorname{Im}[\Delta(y)] \tau_{y},
\end{aligned}
$$

where $m_{\mathrm{e}}$ is the effective electron mass in the 2DEG, $\mu(y)=\mu_{\mathbf{J}} \theta(w / 2-|y|)+\mu_{\mathrm{SC}} \theta(|y|-w / 2)$ is the chemical potential, with $\mu_{\mathbf{J}}\left(\mu_{\mathrm{SC}}\right)$ its value in the junction (below the superconductors), $\alpha$ is the Rashba-spin-orbit-coupling coefficient, $\quad E_{Z}(y)=E_{\mathbf{Z}, \mathbf{J}} \theta(w / 2-|y|)+E_{\mathbf{Z}, \mathrm{SC}} \theta(|y|-w / 2)$
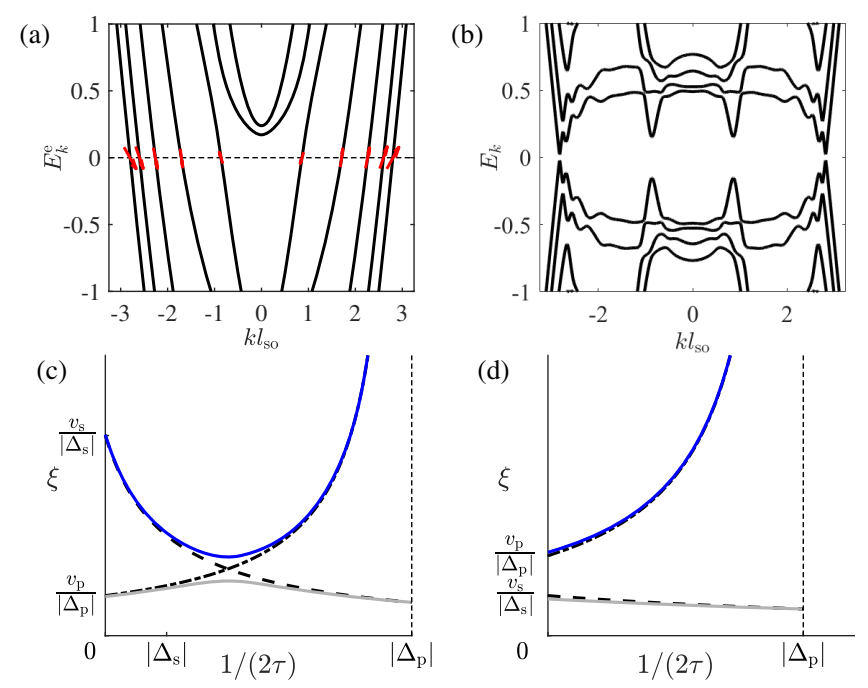

FIG. 3. (a) Example of the low-energy spectrum of the normal strip $(|y|<W / 2)$ before it is coupled to the SCs. Red arrows show the spin expectation value averaged over $y, \int d y\langle\boldsymbol{\sigma}(y)\rangle$. The direction of the arrow indicates the direction of the spin in the (xy) plane. (b) Upon introducing the superconductors, a gap is induced. For the parameters considered here, the junction supports five gapped transverse channels. (c),(d) Localization length versus disorder in a $p$-wave SC $\xi_{\mathbf{p}}$ (black dotted line) and in an $s$-wave SC $\xi_{\mathrm{s}}$ (black dashed line). Disorder increases $\xi_{\mathrm{p}}$ and decreases $\xi_{\mathbf{s}}$. A multichannel TSC can sometimes be viewed as a combination of a $p$-wave SC and an $s$-wave SC. The overall localization length is determined by the larger between $\xi_{\mathrm{p}}$ and $\xi_{\mathrm{s}}$. (c) When the $p$-wave gap $\left|\Delta_{\mathbf{p}}\right|$ exceeds the $s$-wave gap $\left|\Delta_{\mathbf{s}}\right|$, the overall $\xi$ shows nonmonotonic behavior as a function of disorder strength (blue solid line). This is the situation in the planar JJ studied here. (d) When $\left|\Delta_{\mathbf{p}}\right| \leq\left|\Delta_{\mathbf{s}}\right|$, disorder causes $\xi$ to increase monotonically, reaching a phase transition at $1 / 2 \tau_{\mathbf{c}}=\left|\Delta_{\mathbf{p}}\right|$.

is the Zeeman splitting due to the in-plane magnetic field, with $E_{\mathbf{Z}, J}\left(E_{\mathbf{Z}, \mathrm{SC}}\right)$ being its values in the junction (below the superconductors), and $\Delta(y)=\Delta_{0} \theta(|y|-w / 2) \times$ $\exp [i \operatorname{sgn}(y) \phi / 2]$ is the electrons' pairing potential with $\phi$ being the phase difference between the two superconductors. Here, $U(x, y)$ is a random disorder potential having zero average and short-range correlations $\left\langle U(\mathbf{r}) U\left(\mathbf{r}^{\prime}\right)\right\rangle=$ $\delta\left(\mathbf{r}-\mathbf{r}^{\prime}\right) /\left(m_{\mathrm{e}} \tau\right)$, where $\tau$ is the mean free time for disorder scattering in the bare 2DEG. In writing Eq. (1), we have used the Nambu basis $\Psi^{\dagger}(\mathbf{r})=\left[\psi_{\uparrow}^{\dagger}(\mathbf{r}), \psi_{\downarrow}^{\dagger}(\mathbf{r}), \psi_{\downarrow}(\mathbf{r})\right.$, $\left.-\psi_{\uparrow}(\mathbf{r})\right]$, where $\psi_{s}^{\dagger}(\mathbf{r})$ creates an electron in the 2DEG with spin $s$ at position $\mathbf{r}=(x, y)$. Accordingly, the sets of Pauli matrices $\sigma_{\alpha=x, y, z}$ and $\tau_{\alpha=x, y, z}$ operate on the spin and particle-hole degrees of freedom, respectively.

To analyze the disordered system numerically, we use a lattice model and construct a corresponding tight-binding Hamiltonian. The topological invariant $\mathcal{Q}$ and the localization length $\xi$ can be obtained from the scattering matrix between two fictitious leads at $x=0$ and $x=L_{x}$ (which extend throughout the $y$ direction). The scattering matrix is calculated numerically using a recursive Green-function method [27,28]. 
Let $r(\varepsilon)$ be the reflection matrix for electrons and holes incident on the left at energy $\varepsilon$. The topological invariant satisfies [37,38] $\mathcal{Q}=\operatorname{det}[r(\varepsilon=0)]$, which in the limit $L_{x} \rightarrow \infty$ takes the values 1 in the trivial phase and -1 in the topological phase.

We obtain $\xi$ from finite-size scaling of the zero-energy transmission probability matrix $T(0)=\mathbb{1}-r^{\dagger}(0) r(0)$. Except for the phase transition, the eigenvalues of $T(0)$ decay exponentially with $L_{x}[39,40]$. The smallest exponent determines the localization length of midgap zeroenergy states. In the topological phase, this defines the Majorana localization length $\xi$. We average $\xi$ over many disorder realizations.

Figure 1(a) presents the phase diagram of the clean system $[U(x, y)=0]$ previously obtained in Ref. [21]. We note the chemical potential need not be fine-tuned for the system to be topological; in particular, it can be substantially larger than $E_{\mathbf{Z . J}}$. In the topological phase, the junction hosts zero-energy MBS at the junction's ends near $x=0$ and $x=L_{x}$.

Figure 1(b) presents $\xi$ versus disorder strength represented by the inverse mean free time of the underlying $2 \mathrm{DEG} \tau^{-1}$ for different values of $E_{\mathbf{Z}, J}$ and $\phi$ [see markers in Fig. 1(a)]. In all cases shown, $\xi$ first decreases as a function of $\tau^{-1}$, reaching a minimum which can be an order of magnitude smaller than its value in the clean system. This makes the Majorana bound states more protected against perturbations that can potentially couple them. When increasing disorder strength further, $\xi$ eventually increases, diverging at the phase transition to the trivial phase shown by the vertical dashed lines. Notice the phase transition occurs at a critical disorder strength $\tau_{\mathbf{c}}^{-1}$ much larger than $E_{\text {gap }}$.

Low-energy model.-To understand the above results, we consider a more general model of a 1D multichannel TSC comprised of linearly dispersing electronic modes $\phi_{m}(x)$ and given by $H=H_{0}+H_{\text {dis }}$ with

$$
\begin{aligned}
H_{0}= & \sum_{m= \pm 1}^{ \pm N} \int d x\left\{-i v_{m} \phi_{m}^{\dagger}(x) \partial_{x} \phi_{m}(x)\right. \\
& \left.+\frac{1}{2}\left[\Delta_{m} \phi_{m}^{\dagger}(x) \phi_{-m}^{\dagger}(x)+\text { H.c. }\right]\right\}, \\
H_{\mathrm{dis}}= & \sum_{m, n} \int d x e^{i\left(k_{\mathbf{F}, m}-k_{\mathbf{F}, n}\right) x} V_{m n}(x) \phi_{m}^{\dagger}(x) \phi_{n}(x) .
\end{aligned}
$$

Here, each of the $N$ conducting channels contains a rightmoving mode $\left(v_{m>0}>0\right)$ and a left-moving mode $\left(v_{m<0}<0\right), k_{\mathbf{F}, m}$ is the Fermi momentum of the $m$ th mode, $v_{m}$ is the mode velocity, $\Delta_{m}$ is a pairing potential in the $m$ th channel, and $V_{m n}(x)$ are scattering terms arising from disorder. Notice $V_{n m}^{*}(x)=V_{m n}(x)$ due to Hermiticity, and $\Delta_{m}=-\Delta_{-m}$ due to the anticommutativity of $\left\{\phi_{m}\right\}$. In the clean limit, the system is topological for odd $N$ and trivial for even $N$. The Majorana localization length (for odd $N$ ) is determined by the maximal $\xi_{m}^{0}=v_{m} /\left|\Delta_{m}\right|$.
This model can be related to the planar JJ at low energies by first solving the Hamiltonian of Eq. (2) inside the junction $(|y| \leq W / 2)$ in the absence of coupling to the SCs, i.e., when the reflection from the SCs is purely normal [see, e.g., Fig. 3(a)], then linearizing the spectrum near the Fermi points to obtain $\phi_{m}$ and $v_{m}$, and finally considering the induced superconductivity in the form of the pairing potentials $\Delta_{m}$ [28]. This is justified when the Fermi level is far enough from the bottom of the band compared with $\left|\Delta_{m}\right|,\left|V_{m n}\right|$. Omitting interchannel pairings is justified whenever the energy mismatch $\min \left(v_{n}, v_{m}\right)\left|k_{n}-k_{m}\right|$ is large compared with the interchannel pairing.

In the above model, Eq. (2), we assume that $k_{F,-m}=-k_{F, m}, v_{-m}=-v_{m}$, and $V_{m n}=V_{-m,-n}$. This will indeed be the case in the planar $\mathrm{JJ}$ due to a reflection symmetry $\sigma_{x} \mathcal{H}(-x, y) \sigma_{x}=\mathcal{H}(x, y)$ present in the clean limit [28]. The elements of the disorder matrix are normally distributed with zero mean and short-range correlations $\overline{V_{m n}(x) V_{m n}\left(x^{\prime}\right)}=\gamma_{m n} \delta\left(x-x^{\prime}\right)$, where the upper bar denotes disorder averaging, and $\gamma_{m n}$ is related to the disorder-induced transition rate from mode $m$ to $n$ through $\tau_{m n}^{-1}=\left|\gamma_{m n} / v_{n}\right|$. While $\gamma_{m n}$ is generally complex, in our case it may be chosen real and positive, thanks to a timereversal-like symmetry $\mathcal{H}^{*}(x,-y)=\mathcal{H}(x, y)$, which exists in the clean limit $[21,28]$. We make this choice here.

To obtain a correction to $\Delta_{m}$ in the form of a disorder self-energy, we examine the Nambu-Gor'kov Green function $G_{m n}\left(x-x^{\prime} ; i \omega\right)=\int d \tau e^{-i \tau \omega} \overline{\left\langle\mathcal{T}_{\tau} \Phi_{m}\left(x^{\prime}, 0\right) \Phi_{n}^{\dagger}(x, \tau)\right\rangle}$, where $\Phi_{m}^{\dagger}=\left(\phi_{m}^{\dagger}, \phi_{-m}\right)$. In the absence of disorder, the momentum-space Green function reads

$G_{m n}^{0}(q, i \omega=0)=\frac{-\delta_{m n}}{\left(v_{m} q\right)^{2}+\left|\Delta_{m}\right|^{2}}\left(\begin{array}{cc}v_{m} q & \Delta_{m} \\ \Delta_{m}^{*} & -v_{m} q\end{array}\right)$.

For weak disorder, we can obtain the self-energy within the Born approximation [28]

$$
\begin{aligned}
\Sigma_{m}(q, 0) & =\sum_{n \neq m}\left|\gamma_{m n}\right| \int \frac{d p}{2 \pi} e^{i \frac{\alpha_{m n n}}{2} \tau_{z}} \tau_{z} G_{n n}^{0}(p, 0) \tau_{z} e^{-i \frac{\alpha_{m n}}{2} \tau_{z}} \\
& =\sum_{n \neq m} \frac{1}{2 \tau_{m n}} e^{i\left[\arg \left(\Delta_{n}\right)+\alpha_{m n}\right] \tau_{z}} \tau_{x},
\end{aligned}
$$

where $\alpha_{m n} \equiv \arg \left(\gamma_{m n}\right)$. Comparing with the unperturbed Green function, we see that disorder changes the effective pairing potentials according to

$$
\Delta_{m}^{\mathrm{eff}}=\Delta_{m}+\frac{1}{2} \sum_{n \neq m} \frac{1}{\tau_{m n}} e^{i\left[\arg \left(\Delta_{n}\right)+\alpha_{m n}\right]} .
$$

Notice that the contribution of mode $n$ to $\left|\Delta_{m}\right|$ depends on the interchannel scattering rate $\tau_{m n}^{-1}$, the scattering phase $\alpha_{m n}$, and on the relative phase between $\Delta_{m}$ and $\Delta_{n}$. Importantly, disorder can either decrease or increase 
$\left|\Delta_{m}\right|$ (and therefore, increase or decrease $\xi_{m}$ ). The process underlying Eq. (5) is depicted in Fig. 2.

Disordered s-wave vs disordered p-wave superconductor.-We explore two special cases of the multichannel superconductor: (i) a single-channel $p$-wave SC and (ii) a single-(spinful)-channel $s$-wave SC. These cases clarify the nonmonotonic behavior of $\xi$ for the disordered planar JJ observed in Fig. 1(b).

The low-energy Hamiltonian of a single-channel $p$-wave SC is obtained by setting $N=1$ in Eq. (2), with $v_{1} \equiv v_{\mathbf{p}}$, $\Delta_{1}=-\Delta_{-1} \equiv \Delta_{\mathbf{p}}$, and $\tau_{1,-1} \equiv \tau_{\mathbf{p}}$. Equation (5) then yields

$$
\left|\Delta_{\mathbf{p}}^{\mathrm{eff}}\right|=\left|\Delta_{\mathbf{p}}\right|-1 / 2 \tau_{\mathbf{p}} .
$$

The localization length can then be obtained by $\xi_{\mathbf{p}}=v_{\mathbf{p}} /\left|\Delta_{\mathbf{p}}^{\text {eff }}\right|$ yielding the known result [9] $1 / \xi_{\mathbf{p}}=$ $1 / \xi_{\mathbf{p}}^{0}-1 / 2 l_{\mathbf{p}}$, where $l_{\mathbf{p}}=v_{\mathbf{p}} \tau_{\mathbf{p}}$ is the mean free path and $\xi_{\mathbf{p}}^{0}=v_{\mathbf{p}} /\left|\Delta_{\mathbf{p}}\right|$.

For a single-channel $s$-wave superconductor, there are no zero-energy end modes, and $\xi$ is the length to which a single electron at zero energy penetrates the superconductor before being reflected. The index $m=1,2$ corresponds to the two spin directions. The spin-singlet nature of the pairing dictates $\Delta_{1}=-\Delta_{2}=\Delta_{\mathrm{s}}$, and the spin independence of the disorder forbids intrachannel scattering and dictates $\tau_{1,-1}^{-1}=\tau_{2,-2}^{-1}=0$. Furthermore, the two velocities are the same $v_{1}=v_{2}=v_{\mathrm{s}}$.

Setting this in Eq. (5), we have

$$
\left|\Delta_{\mathbf{s}}^{\mathrm{eff}}\right|=\left|\Delta_{\mathbf{s}}\right|+1 / 2 \tau_{\mathbf{s}}^{-1},
$$

where $\tau_{11}=\tau_{22} \equiv \tau_{\mathrm{s}}, \quad$ and correspondingly, $1 / \xi_{\mathrm{s}}=$ $1 / \xi_{\mathrm{s}}^{0}+1 / 2 l_{\mathrm{s}}$, where $l_{\mathrm{s}}=v_{\mathrm{s}} \tau_{\mathrm{s}}$. Unlike the case of the single-channel $p$-wave SC, the localization length in the $s$-wave case decreases monotonically. We emphasize that the relative sign difference in Eq. (6) compared to Eq. (7) stems from (i) lack of scattering between opposite spins and (ii) the $s$-wave spin-singlet nature of the pairing. While these results for $\xi_{\mathrm{p}}$ and $\xi_{\mathrm{s}}$ were obtained using a weakdisorder perturbative analysis, they are actually exact for the linearized model of Eq. (2), as shown in the Supplemental Material [28].

The results for $\xi_{\mathrm{p}}$ and $\xi_{\mathrm{s}}$ let us understand the nonmonotonic behavior of $\xi$ in the planar JJ [see Fig. 1(b)]. The low-energy spectrum of subgap excitations confined between the two superconductors may be seen as coming out of superconducting pairing of several low-energy modes. Figure 3(a) presents an example of the spectrum of the Hamiltonian Eq. (1) confined within the junction under the assumption of full normal reflection. The red arrows representing the spin expectation values of the modes indicate that the outer channels (larger Fermi momentum) are largely spin polarized due to the spinorbit coupling, with opposite-momentum modes having approximately opposite spins. For the inner channel, the spin varies along the $y$ direction, resulting in a smaller expectation value.

Since pairing is induced by an s-wave SC, the largemomentum channels (being spin polarized) behave as an $s$-wave SC, with a localization length $\xi_{\mathrm{s}}$ that decreases with disorder. In contrast, the small-momentum channel is not spin polarized and allows for intrachannel backscattering. Consequently, this channel behaves as a disordered $p$-wave $\mathrm{SC}$, with localization length $\xi_{\mathrm{p}}$ that increases with disorder. The overall localization length of the system $\xi$ is then the larger between $\xi_{\mathbf{s}}$ and $\xi_{\mathbf{p}}$.

The behavior of $\xi$ versus disorder therefore depends on the relative size of $\left|\Delta_{\mathbf{p}}\right|$ and $\left|\Delta_{\mathbf{s}}\right|$. Assuming for simplicity, $\tau_{\mathbf{p}} \sim \tau_{\mathbf{s}} \sim \tau$ and $v_{\mathbf{s}} \sim v_{\mathbf{p}}$, we find that when $\left|\Delta_{\mathbf{p}}\right|>\left|\Delta_{\mathbf{s}}\right|$ [Fig. 3(c)], the localization length decreases for weak disorder. With stronger disorder, the two gaps approach one another. Consequently, scattering between the largemomentum and the low-momentum channels causes "level repulsion" between $\xi_{\mathrm{s}}$ and $\xi_{\mathrm{p}}$, as depicted in Figs. 3(c) and 3(d), and $\xi$ increases with disorder (blue solid line). Notice that the critical disorder strength $1 / 2 \tau_{\mathbf{c}}=\left|\Delta_{\mathbf{p}}\right|$ can be much larger than the gap of the clean system $\mid \Delta_{\mathbf{s}}$ I. In contrast, if $\left|\Delta_{\mathbf{p}}\right| \leq\left|\Delta_{\mathbf{s}}\right|[$ [Fig. 3(d)], disorder causes $\xi$ to increase monotonically, diverging at the critical disorder $1 / 2 \tau_{\mathbf{c}}=\left|\Delta_{\mathbf{p}}\right|$, which now equals the gap of the clean system.

In the planar JJ, the gap of the large-momentum channels is indeed the smaller one [see Fig. 3(b)]. For a not-toonarrow junction, the low-momentum gap is approximately $\min \left(\Delta_{0}, h v_{\mathbf{F}} / W\right)$, while the large-momentum gap is approximately $\hbar^{2} / 2 m_{\mathrm{e}} W^{2}$. This difference may be viewed
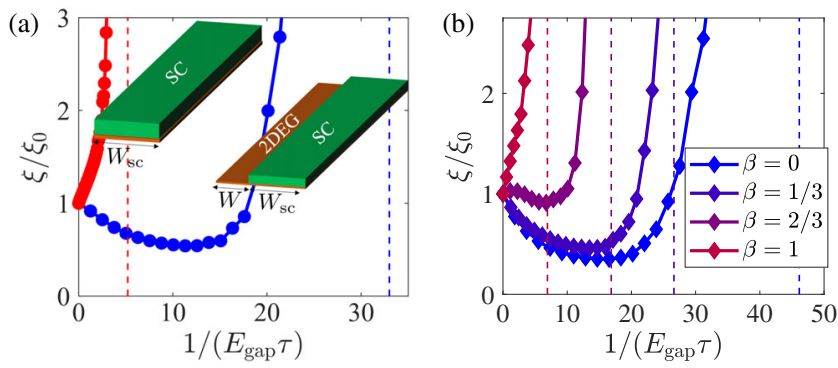

FIG. 4. (a) Majorana localization length versus disorder in a 2DEG coupled to an $s$-wave SC in two different geometries. In the first geometry (blue), the smaller superconducting gap is at the large-momentum channels, while in the second geometry (red), the gaps of the different channels are of the same order. The system's parameters are as in Fig. 1, with $E_{\mathbf{Z}, \mathbf{J}}=1$ and with $\Delta_{0}=1\left(\Delta_{0}=0.25\right)$ for the first (second) geometry (the reduced $\Delta_{0}$ in the second geometry is necessary for the system to be topological). (b) Majorana localization length for the planar JJ [see Eq. (1) and Fig. 1] for different ratios of magnetic-disorder strength to potential-disorder strength. Magnetic disorder couples opposite-spin modes. The rest of the system's parameters are as in Fig. 1, with $\phi=\pi, E_{\mathbf{Z} . \mathbf{J}}=1$. 
as originating from the fact that high-momentum electrons propagate almost parallel to the SCs, and are therefore only weakly coupled to the SCs. For this system, then, disorder may increase the effective gap from the scale of $\hbar^{2} / 2 m_{\mathbf{e}} W^{2}$ to the scale of $\Delta_{0}$.

We test our understanding by studying two geometries of superconducting proximity [Fig. 4(a)], where a strip of 2DEG is coupled to a single SC from the side (blue line) or from above the strip (red line). While in the former the large-momentum ( $s$-wave) gap is the smallest, giving rise to behavior similar to the planar JJ, this is not the case in the latter geometry, resulting in a monotonically increasing $\xi$.

As another test, we add to Eq. (1) a magnetic disorder term $\mathcal{H}_{\mathbf{m}}=U_{\mathbf{m}}(\boldsymbol{r}) \sigma_{z}$. Here, $U_{\mathbf{m}}(\boldsymbol{r})$ is a random field with zero average and correlations $\left\langle U_{\mathbf{m}}(\boldsymbol{r}) U_{\mathbf{m}}\left(\boldsymbol{r}^{\prime}\right)\right\rangle=$ $\gamma_{\mathbf{m}} \theta(W / 2-|y|) \delta\left(\boldsymbol{r}-\boldsymbol{r}^{\prime}\right)$ [41]. Figure 4(b) presents $\xi$ for different values of the ratio between magnetic and potential disorder $\beta=\gamma_{\mathbf{m}} / \gamma$, where $\gamma=1 /\left(m_{\mathbf{e}} \tau\right)$. Since magnetic disorder can scatter between the opposite-spin states, the large-momentum channels do not behave anymore as an $s$ wave SC, and instead they are more similar to a multichannel $p$-wave SC [15-17]. Indeed, with increasing $\beta$, the disorder-induced decrease in $\xi$ diminishes.

We have benefited from the insightful comments of B. Halperin and Y. Oreg. We also thank M. Buchhold for useful discussions. We acknowledge support from the Walter Burke Institute for Theoretical Physics at Caltech (A. H.), the Israel Science Foundation (A. S.), the European Research Council under the European Community Seventh Framework Program (Grant No. FP7/2007-2013/ MUNATOP) (A. S.), Microsoft Station Q (A. S.), and the DFG (CRC/Transregio 183, Grant No. EI 519/7-1) (A. S.).

[1] A. Kitaev, Phys. Usp. 44, 131 (2001).

[2] N. Read and D. Green, Phys. Rev. B 61, 10267 (2000).

[3] X.-L. Qi and S.-C. Zhang, Rev. Mod. Phys. 83, 1057 (2011).

[4] J. Alicea, Rep. Prog. Phys. 75, 076501 (2012).

[5] C. W. J. Beenakker, Annu. Rev. Condens. Matter Phys. 4, 113 (2013).

[6] R. Lutchyn, E. Bakkers, L. Kouwenhoven, P. Krogstrup, C. Marcus, and Y. Oreg, Nat. Rev. Mater. 3, 52 (2018).

[7] R. Aguado, La Rivista Del Nuovo Cimento 40, 523 (2017).

[8] O. Motrunich, K. Damle, and D. A. Huse, Phys. Rev. B 63, 224204 (2001).

[9] P. W. Brouwer, M. Duckheim, A. Romito, and F. von Oppen, Phys. Rev. Lett. 107, 196804 (2011).

[10] A. M. Lobos, R. M. Lutchyn, and S. Das Sarma, Phys. Rev. Lett. 109, 146403 (2012).

[11] F. Pientka, A. Romito, M. Duckheim, Y. Oreg, and F. von Oppen, New J. Phys. 15, 025001 (2013).

[12] D. A. Huse, R. Nandkishore, V. Oganesyan, A. Pal, and S. L. Sondhi, Phys. Rev. B 88, 014206 (2013).

[13] I. Adagideli, M. Wimmer, and A. Teker, Phys. Rev. B 89, 144506 (2014).
[14] This result is valid when the Fermi energy is large compared with $E_{\text {gap }}$, which is the limit of interest here. For the opposite limit, see Ref. [11].

[15] A. C. Potter and P. A. Lee, Phys. Rev. Lett. 105, 227003 (2010).

[16] M.-T. Rieder, P. W. Brouwer, and I. Adagideli, Phys. Rev. B 88, 060509 (2013).

[17] M.-T. Rieder and P. W. Brouwer, Phys. Rev. B 90, 205404 (2014).

[18] B. Lu, P. Burset, Y. Tanuma, A. A. Golubov, Y. Asano, and Y. Tanaka, Phys. Rev. B 94, 014504 (2016).

[19] P. Burset, B. Lu, S. Tamura, and Y. Tanaka, Phys. Rev. B 95, 224502 (2017).

[20] M. Hell, M. Leijnse, and K. Flensberg, Phys. Rev. Lett. 118, 107701 (2017).

[21] F. Pientka, A. Keselman, E. Berg, A. Yacoby, A. Stern, and B. I. Halperin, Phys. Rev. X 7, 021032 (2017).

[22] M. Hell, K. Flensberg, and M. Leijnse, Phys. Rev. B 96, 035444 (2017).

[23] S. Hart, H. Ren, M. Kosowsky, G. Ben-Shach, P. Leubner, C. Brüne, H. Buhmann, L. W. Molenkamp, B. I. Halperin, and A. Yacoby, Nat. Phys. 13, 87 (2017).

[24] P. W. Brouwer, M. Duckheim, A. Romito, and F. von Oppen, Phys. Rev. B 84, 144526 (2011).

[25] H. Ren, F. Pientka, S. Hart, A. Pierce, M. Kosowsky, L. Lunczer, R. Schlereth, B. Scharf, E. M. Hankiewicz, L. W. Molenkamp et al., arXiv:1809.03076.

[26] A. Fornieri, A. M. Whiticar, F. Setiawan, E. P. Marín, A. C. Drachmann, A. Keselman, S. Gronin, C. Thomas, T. Wang, R. Kallaher et al., arXiv:1809.03037.

[27] P. A. Lee and D. S. Fisher, Phys. Rev. Lett. 47, 882 (1981).

[28] See Supplemental Material at http://link.aps.org/ supplemental/10.1103/PhysRevLett.122.126801 for details on (i) numerical simulations, (ii) analysis of the low-energy model, and (iii) calculation of the localization length in the case of a single-channel $p$-wave and $s$-wave SCs, which includes Refs. [29-36].

[29] D. S. Fisher and P. A. Lee, Phys. Rev. B 23, 6851 (1981).

[30] S. Iida, H. A. Weidenmüller, and J. Zuk, Ann. Phys. (N.Y.) 200, 219 (1990).

[31] A. C. Potter and P. A. Lee, Phys. Rev. B 83, 094525 (2011).

[32] J. Bardeen, Phys. Rev. Lett. 6, 57 (1961).

[33] A. Haim, K. Wölms, E. Berg, Y. Oreg, and K. Flensberg, Phys. Rev. B 94, 115124 (2016).

[34] B. I. Halperin, Advance in Chemical Physics, edited by I. Prigogine (John Wiley \& Sons, Inc., New York, 1967).

[35] O. Dorokhov, JETP Lett. 36, 318 (1982).

[36] P. Mello, P. Pereyra, and N. Kumar, Ann. Phys. (N.Y.) 181, 290 (1988).

[37] A. R. Akhmerov, J. P. Dahlhaus, F. Hassler, M. Wimmer, and C. W. J. Beenakker, Phys. Rev. Lett. 106, 057001 (2011).

[38] I. C. Fulga, F. Hassler, A. R. Akhmerov, and C. W. J. Beenakker, Phys. Rev. B 83, 155429 (2011).

[39] C. W. J. Beenakker, Rev. Mod. Phys. 69, 731 (1997).

[40] F. Evers and A. D. Mirlin, Rev. Mod. Phys. 80, 1355 (2008).

[41] We limit $U_{\mathbf{m}}(\boldsymbol{r})$ to the junction since we are not interested here in its effect on the SCs. 